\title{
Criminologie
}

\section{Quand les femmes sont victimes..., quand les hommes appliquent la loi}

\section{Micheline Baril, Marie-Marthe Cousineau et Sylvie Gravel}

Volume 16, numéro 2, 1983

Les femmes et la justice pénale

URI : https://id.erudit.org/iderudit/017183ar

DOI : https://doi.org/10.7202/017183ar

Aller au sommaire du numéro

Éditeur(s)

Les Presses de l'Université de Montréal

ISSN

0316-0041 (imprimé)

1492-1367 (numérique)

Découvrir la revue

Citer cet article

Baril, M., Cousineau, M.-M. \& Gravel, S. (1983). Quand les femmes sont victimes..., quand les hommes appliquent la loi. Criminologie, 16(2), 89-100. https://doi.org/10.7202/017183ar d'utilisation que vous pouvez consulter en ligne. 


\section{QUAND LES FEMMES SONT VICTIMES... QUAND LES HOMMES APPLIQUENT LA LOI... Micheline Baril*, Marie-Marthe Cousineau**, Sylvie Gravel**}

Durant la dernière décade, une multitude de recherches et de publications sont apparues sur un problème jusque-là considéré comme innommable: la violence exercée contre une femme à l'intérieur d'une relation de couple hétérosexuel. Ainsi, une revue non exhaustive de la littérature, entreprise par The National Clearinghouse on Family Violence aux États-Unis, recensait plus de mille titres entre les années 1970 et 1979.

Pourtant, en dépit de cette soudaine abondance d'écrits, il nous faut constater que les femmes continuent d'être agressées à l'intérieur même du sanctuaire où elles se réfugient, du foyer qu'elles créent. L'appareil de justice ne sait comment les protéger.

La mentalité qui fait des femmes des êtres faibles et vulnérables attribue à l'homme un rôle de protecteur. Dès lors, la société, à commencer par les victimes mêmes, ne peut tolérer une situation aussi menaçante, où le protecteur devient l'agresseur, où le foyer se transforme en terrain de bataille, où la vie de couple aboutit à un régime de terreur. La victime a trop souvent dû jouer l'autruche; la société a publiquement fermé les yeux devant ces drames intimes (pour peu, on dirait, érotiques).

L'absence de statistiques a favorisé l'incrédulité, le mutisme et l'inaction. Mythes et contre-mythes, attestations et démentis, se succèdent sans que jamais on ne puisse décrire la violence conjugale dans ses dimentions chiffrables. Encore en 1983, il existe très peu de données sur l'ampleur du problème dans un territoire donné et encore moins d'études évaluatives de l'efficacité de diverses mesures de protection. Stigmatisante pour les victimes, la violence dans le couple est peu dénoncée. De plus, aucune des agences auxquelles cette violence est parfois rapportée, autorités médicales, services sociaux, police et tribunaux, ne tient de dossiers permettant de dénombrer les cas d'agressions dans le couple, et de les connaître

\footnotetext{
* Professeur adjoint à l'École de criminologie, Université de Montréal.

** Assistante de recherche.
} 
sous divers aspects. Consulter les archives de ces agences pour y répérer les voies de fait entre conjoints requiert un travail de bénédictin et une boule de cristal.

La pauvreté des connaissances demeure donc le principal obstacle à la protection des femmes dans leur foyer. La réaction sociale en est un second. Hommes et femmes sont menacés dans leur sécurité, leur identité, leur vie émotive, par un tel phénomène même s'ils n'en ont eu aucune expérience personnelle. Une femme qui avait survécu à une tentative de meurtre de la part de son conjoint exprimait ainsi cette menace :

Les hommes, c'est comme si on les attaquait personnellement de leur raconter ce qu'on a subi. J'ai senti ça.

Suite à l'agression, cette femme n'avait eu affaire qu'à des hommes : médecins, policiers, avocats, juges. C'est d'ailleurs le lot de la plupart des femmes agressées par un conjoint.

\section{QUELQUES SOURCES DE DONNÉES}

Afin d'établir les balises d'une action préventive, un programme de recherches a été entrepris à Montréal, qui veut mieux cerner le problème, la réaction sociale formelle et informelle et, éventuellement, évaluer systématiquement les mesures sociales, judiciaires ou para-judiciaires. L'étude a pris naissance en 1979, avec Sheilagh Hodgins et Ginette Larouche de l'École de service social de l'Université de Montréal. Depuis, d'autres études ont été réalisées. Décrivons brièvement deux d'entre elles.

La première voulait mesurer l'ampleur de la violence conjugale dénoncée à la police de la Communauté urbaine de Montréal en 1980 , décrire cette violence et ses protagonistes et examiner la réaction de la police. Un relevé systématique de tous les dossiers de voies de fait ouverts dans les vingt-quatre districts de police a révélé l'existence de 777 cas d'agressions entre conjoints. Dans tous les cas, sauf un, la victime était une femme. Les dossiers d'enquête ont aussi été consultés pour 239 des 777 cas identifiés (Baril, M., Cousineau, M-M., Gravel, S., 1983).

La seconde recherche s'inscrit en continuité de la précédente. Il s'agissait d'abord de trouver, parmi les quelque vingt mille dossiers ouverts à la Cour municipale de Montréal en 1980, ceux qui concernent les femmes violentées par leur conjoint et d'étudier la réaction judiciaire à ces situations. Le relevé des données pertinentes dans les dossiers était complété par des entrevues formelles non structurées, 
auprès des juges, des procureurs de la Couronne et des avocats de pratique criminelle ou civile. Nous avons pu, également, réaliser plusieurs observations dans les salles d'audience, dans les corridors et à la Chambre des plaintes. Cette étude a révélé l'existence de 367 causes entendues à la Cour municipale concernant des agressions de la part d'un conjoint pour l'année en cause.

Dans les deux études, les meurtres et les tentatives de meurtre ont été exclus, de même que tous les cas où la relation entre agresseur et victime ne relevait pas très clairement d'un lien de couple.

\section{LES FEMMES VIOLENTÉES}

Le profil socio-démographique des Montréalaises qui ont fait appel à l'appareil de justice en 1980, suite à une violence de leur conjoint, diffère peu de celui de la femme québécoise. Maints chercheurs l'ont écrit avant nous : la femme battue n'a pas de profil distinct.

Comme l'ensemble des femmes, elle vit le plus souvent dans une situation de dépendance économique : elle n'a pas d'occupation rémunérée ou occupe un emploi subalterne. Mais on retrouve des femmes battues également chez les cadres et les professionnelles.

Comparativement à l'ensemble de la population féminine du Québec, les femmes battues des deux populations étudiées sont plus jeunes et vivent plus souvent en union de fait.

Si on compare les femmes qui ont été écoutées par la police et celles qui ont fait appel au tribunal, on constate qu'elles ont moins de quarante ans dans les trois quart des cas rapportés à la police et dans les deux tiers des cas portés devant le tribunal. Apparemment, les jeunes femmes seraient plus souvent agressées mais auraient moins tendance à poursuivre jusqu'au bout la démarche amorcée par l'appel à la police. Les policiers prennent aussi moins au sérieux les querelles domestiques chez les jeunes couples. En vieillissant, à cause de la répétition ou de l'aggravation de la violence et des enfants qui ont grandi, les femmes optent davantage pour la poursuite judiciaire devant un tribunal de juridiction crimineile.

Les deux études démontrent que, contrairement aux croyances populaires, les femmes se libèrent des unions violentes mais ne sont pas nécessairement protégées pour autant. Chez les femmes qui ont appelé la police, on a constaté que $28 \%$ d'entre elles avaient cessé de vivre une relation de couple avec l'agresseur au moment où s'est pro- 
duite l'agression. Cette proportion est de $60 \%$ chez celles qui se sont rendues au tribunal.

Par ailleurs, les agressions physiques les plus violentes semblent se produire entre personnes qui cohabitent. En quittant le domicile conjugal, la femme crée une certaine barrière entre elle et son conjoint agressif qui la tient à l'écart des coups, même si elle ne la libère pas du harcèlement ni de la menace. Ainsi, on observe que $72 \%$ des femmes ne vivaient plus avec leur agresseur au moment où le tribunal a été saisi de la plainte et que près de $45 \%$ d'entre elles faisaient état de diverses formes de menaces et de harcèlement plutôt que de coups et blessures. Menaces de mort, de sévices corporels, menaces d'enlever les enfants, rôdage incessant autour du domicile de la plaignante, introduction par effraction chez-elle, voilà la nature de la plupart des griefs dans lesquels l'agression physique n'a pas été invoquée.

\section{LA RÉACTION DE L'APPAREIL POLICIER}

Le Service de police de la Communauté urbaine de Montréal a reçu, en 1980, un nombre indéterminé d'appels à l'aide concernant des situations de violence conjugale. Il a donné suite à ces appels, rédigé un rapport et qualifié l'événement de voies de fait contre la conjointe dans 776 cas.

a) L'APPEL À LA POLICE

Les femmes violentées n'appellent pas toutes la police. Dans bien des cas, les voisins, les enfants ou d'autres membres de l'entourage en prennent l'initiative. Les appels sont reçus et triés par des répartiteurs civils qui disposent de peu de critères pour juger de l'urgence ou de la gravité de la situation. L'appel lui-même est souvent ambigu et la description des faits incomplète.

Au moment de l'étude, il n'existait aucune consigne claire de priorité au Service de police, concernant les situations de violence conjugale mais la direction du service examinait la question afin de prévenir une escalade de ces violences et leur aboutissement en homicides.

Nos données étant recueillies dans les dossiers, nous ignorons donc dans quelle mesure les policiers peuvent négliger de répondre à un appel ou de rédiger un rapport d'événement.

b) UNE ÉVALUATION DE LA GRAVITÉ DE LA SITUATION

Au Service de Police de la Communauté urbaine de Montréal, toutes les voies de fait sont classées indifféremment, qu'elles se 
soient produites ou non entre les membres d'une famille. En 1980 , $12,4 \%$ des 6245 plaintes pour voies de fait enregistrées concernaient plus précisément des cas de violence envers une conjointe. De ce nombre, 656 rapports $(84,5 \%)$ ont été classés parmi les voies de fait simples, alors que seulement $120(15,5 \%)$ se trouvaient regroupés sous la rubrique voies de fait graves. Bien que cette tendance à classer selon le moindre mal soit généralisé à l'ensemble des voies de fait dénoncées, elle semble s'accentuer lorsque celles-ci se produisent entre conjoints.

$\mathrm{Ni}$ la gravité des blessures ou leur nature ni les circonstances de la victimisation (lieu, présence de témoins, grossesse de la femme), ni l'histoire de violence, ni le type de liens unissant les protagonistes, ne permettent de prédire le chef d'accusation qui sera privilégié par la police. L'analyse des dossiers n'a révélé aucun critère prépondérant d'évaluation de la gravité des situations.

Les décisions se prennent semble-t-il, de façon assez spontanée et intuitive où le facteur humain tient une place considérable. L'évaluation des policiers est largement tributaire d'une négociation de plainte entre eux et la victime. Les commentaires contenus dans les rapports d'événements laissent croire que les policiers sont influencés par les intentions exprimées par la victime.

On remarque aussi que les cas ne sont pas répartis uniformément sur le territoire de la Communauté urbaine de Montréal et que, dans certains districts, on a plus tendance à les considérer comme des agressions graves. De façon générale, le nombre de plaintes enregistrées et la façon de les classifier semble réfléter davantage les pratiques adoptées par les divers postes de police que la distribution réelle du phénomène à travers le territoire.

En définitive, dès le début du processus, la police prend une décision quand à l'importance de l'affaire. Cette décision est fondée sur une appréciation bien subjective des intentions et de la crédibilité de la victime et semble relever davantage d'un souci d'efficacité administrative que d'un désir de protection. Mais, cette première décision aura une influence sur les étapes subséquentes.

\section{c) L'INTERVENTION AUPRÈS DE L'AGRESSEUR}

Les policiers ont procédé à l'arrestation de l'agresseur dans $10 \%$ des cas. Si on fait exception de quelques cas d'expulsion du conjoint ou de saisie d'armes, c'est la seule intervention des policiers auprès des suspects. Il faut dire que celui-ci avait souvent quitté les lieux avant l'arrivée de la police. 
Ce faible taux d'arrestation permet difficilement d'isoler les critères de décision de la police. Il y a plus souvent arrestation quand l'événement est défini comme voies de fait graves et les policiers sont plus réticents à intervenir si les conjoints habitent ensemble. Que l'agresseur possède ou non un casier judiciaire, la décision n'en semble pas influencée.

Les policiers éprouvent certaines difficultés à interpréter la loi et leurs pouvoirs en matière d'arrestation de conjoints abusifs. D'une part, ils disent ne pas disposer de critères précis pour évaluer la gravité de l'acte et d'autre part, ils semblent préoccupés par les risques de faire l'objet d'une poursuite civile en cas d'arrestation non justifiée. Selon eux, il s'agirait également d'une des situations les plus dangereuses auxquelles ils aient à faire face dans l'exercice de leur profession.

\section{d) L'INTER VENTION AUPRES DE LA VICTIME}

Dans plus de la moitié des cas, les policiers n'interviennent pas auprès de la victime. Ils ont conduit $27 \%$ des plaignantes dans un lieu d'hébergement ou de traitement, le plus souvent à l'hôpital. Seulement dix femmes ont été escortées vers une maison pour femmes en difficulté. Les policiers connaissent sans doute très mal cette ressource, laquelle, d'ailleurs, ne suffit pas à répondre aux demandes.

Quelques femmes (12\%) se sont rendues à l'hôpital ou dans une maison d'hébergement par leurs propres moyens, mais parfois sur le conseil des policiers et une sur cinq s'est refugiée chez des amis ou des membres de sa famille.

Les pratiques d'intervention varient selon les postes de police. Dans les régions sud, centre et nord, environ $30 \%$ des cas signalés résultent dans une action concrète de la police auprès de la victime. $\mathrm{Ce}$ pourcentage augmente considérablement dans la région ouest $(55 \%)$, plus favorisée sur le plan socio-économique, où les victimes sont en principe mieux informées et disposent de plus de moyens. Par contre, dans l'est de la ville, là où le phénomène des femmes battues serait fréquent, là où les parties sont relativement démunies, l'assistance de la police serait à son plus bas niveau : seulement $18 \%$ des plaignantes auraient été aidées directement ou indirectement.

La gravité perçue de la victimisation influence la décision des policiers d'intervenir ou non. Ainsi, il y a assistance dans près des trois quarts des assauts définis comme graves comparativement à $28 \%$ de ceux qui sont qualifiés de mineurs. 
Dans $32 \%$ des rapports d'événements, les policiers ont mentionné avoir avisé la plaignante des mesures à prendre ou l'avoir informée de ses droits. Dans la grande majorité de ces cas, on ferme le dossier et on laisse la plaignante se débrouiller et faire elle-même les démarches.

Parfois, les policiers disent à la victime que, si elle désire porter plainte devant le tribunal, elle doit communiquer avec tel détective. Généralement, et de façon systématique dans certains postes de police, les policiers avisent la plaignante d'aller porter plainte à la Cour municipale. Or, précisément depuis janvier 1980, il a été convenu entre le S.P.C.U.M. et la Cour municipale que les policiers doivent acheminer toutes leurs plaintes et que la Cour ne reçoit plus directement les plaignants. C'est done un lourd fardeau que les policiers donnent aux victimes qui doivent dorénavant insister pour être entendues à la Cour et qui seront, souvent, retournées à la police pour obtenir un rapport d'enquête.

\section{e) LES MESURES ENVISAGÉES PAR LA PLAIGNANTE}

Si les policiers n'interviennent pas davantage, c'est peut-être qu'ils sont persuadés que les victimes ne désirent pas procéder. À l'inverse, les victimes restent peut-être inactives faute de l'appui des policiers.

Dans $32 \%$ des dossiers, les intentions de la victime n'ont malheureusement pas été notées. Par ailleurs, $42 \%$ des femmes ont informé le policier qu'elles prendraient des mesures pour remédier à leur situation. La plupart d'entre elles projetaient loger une plainte devant un tribunal de juridiction criminelle. Plusieurs étaient indécises $(19 \%)$ et $7 \%$ ne désiraient pas prendre de mesures.

Les suggestions, les conseils, les informations donnés par les policiers influencent les décisions des femmes. La plupart de celles qui refusent quelque mesure que ce soit n'ont pas été guidées par les policiers. Par contre, celles qui ont reçu des renseignements décident en majorité d'entreprendre des poursuites au criminel ou au civil. Peut-être ignoraient-elles auparavant les recours disponibles. Peutêtre interprètent-elles ces suggestions comme une marque d'attention, un appui, la confirmation que leur situation n'est pas tolérable et qu'elles sont justifiées de vouloir la corriger.

Les études sur la violence conjugale mentionnent très souvent le grand isolement de la femme qui est battue, isolement explicable en partie par l'insensibilité des institutions à son égard. La légitimisation implicite de la violence conjugale par la société porte la femme 
battue à accepter sa situation puisque personne ne semble en reconnaître la gravité. Alors, quand des policiers semblent porter un certain intérêt à leur situation, elles se sentent un peu plus en droit de prendre des mesures. Même si par la suite, comme nous le verrons plus tard, elles peuvent revenir sur leur décision.

\section{f) DE LA POLICE AU TRIBUNAL}

Après avoir jugé de la gravité de la situation et décidé qu'un rapport doit être fait, deux possibilités s'offrent au policier: il peut classer la plainte ou recommander une enquête. En principe, lorsqu'un suspect est identifié, une enquête devrait être recommandée. Or, dans les situations de femmes battues, le suspect est toujours identifié. Il n'est donc pas étonnant que dans près des trois quarts des cas ( $84 \%$ si les voies de fait ont été jugées graves), une enquête ait été recommandée. Les intentions de la plaignante concernant sa protection future ont ici beaucoup d'impact sur la décision de la police. Lorsque la victime fait savoir qu'elle désire intenter des poursuites, l'enquête est requise dans $81 \%$ des cas comparativement à $46 \%$ lorsqu'elle n'a pas manifesté une telle volonté.

Comme le policier patrouilleur, l'enquêteur porte un jugement sur la plainte et décide de la classer ou de porter une accusation. Environ $32 \%$ des cas se sont soldés par une mise en accusation, proportion vraiment très minime étant donné que l'identité du suspect est toujours connue. Les enquêtes sont souvent effectuées de façon très sommaire et il semble, alors, que c'est surtout la volonté de la victime de maintenir sa décision de poursuivre qui incite l'enquêteur à acheminer la plainte devant le tribunal. Lorsque la victime change d'idée, on vérifie rarement si sa décision a été prise librement ou sous la pression de menaces.

Deux autres facteurs influencent l'enquêteur : les antécédents judiciaires et l'arrestation de l'agresseur. Ainsi, dans $64 \%$ des cas où le suspect possède un dossier judiciaire, l'enquête se termine par une mise en accusation comparativement à $21 \%$ des cas où l'agresseur n'a jamais eu affaire à la justice. De même, s'il y a eu arrestation, $56 \%$ des plaintes sont acheminées vers le tribunal contre $28 \%$ en l'absence d'arrestation.

\section{LA RÉACTION DE L'APPAREIL JUDICIAIRE}

Dans la Communauté urbaine de Montréal, les causes de violence conjugale sont généralement entendues dans les Cours municipales qui ont juridiction. La plus importante est la Cour municipale 
de Montréal où nous avons relevé 367 causes de cette nature en 1980. Une rapide enquête téléphonique suggère qu'environ 150 causes auraient été portées à l'attention des autres Cours municipales durant la même année. Un nombre indéterminé de plaintes est acheminé vers la Cour des Sessions de la Paix.

\section{a) LA DÉNONCIATION}

On peut constater, en premier lieu, que les tribunaux traitent plus de plaintes que les policiers ne leur en transmettent.La situation est assez surprenante dans un système judiciaire qui laisse très peu de place à la plainte privée. On aurait pu s'attendre à environ 250 poursuites sur la foi des dossiers de la police mais il y en a eu au moins 600 .

À la Cour municipale de Montréal où s'est déroulée l'étude, en dépit de l'entente intervenue entre la police et la Cour, le tiers des dénonciations ont été faites directement par la victime et il semble que dans la majorité des cas, la police n'ait jamais ouvert de dossier. Comme l'affirmait un des juges interviewés,

Les plaintes qu'on dit privées sont très particulières aux conflits dans lesquels les parties se connaissent. C'est difficile d'imaginer un vol à main armée où le citoyen victime, la caissière par exemple, irait lui-même signer une dénonciation devant le procureur ou le juge de paix.

Le «caractère privé» de la violence dans le couple est en quelque sorte entériné par l'appareil de justice. Mais alors, si les voies de fait infligées à un conjoint sont du domaine privé, des torts causés à un individu par un autre individu, ne devraient-ils pas relever de la juridiction civile plutôt que de l'instance criminelle?

Les dénonciations signées par la police proviennent à $27 \%$ d'un seul des vingt-quatre districts de police. Les deux districts adjacents à ce poste sont responsables de 3 et $13 \%$ de l'ensemble des dénonciations et pourtant ils desservent une population très semblable sur le plan socio-démographique. La disparité dans le traitement des plaintes est flagrante.

Le plus souvent, l'avocat de la ville maintient le chef d'accusation proposé par la police. Dans la moitié de ces cas, on a accusé de voies de fait, simples $(42 \%)$ ou graves $(9 \%) ; 28 \%$ des accusations ont été portées en vertu de l'article 745-1 qui prévoit que : «Quiconque craint qu'une autre personne ne cause des lésions personnelles à lui-même, à son épouse ${ }^{1}$ ou à son enfant, ou n'endommage sa pro- 
priété, peut déposer une dénonciation devant un juge de paix"); les autres accusations $(21 \%)$ regroupent des chefs divers : menaces par lettres ou appels téléphonique, troubler la paix, entrave à la justice, bruit, etc.

Lorsque l'accusation portée ne correspond pas à la description des faits signée par le policier ou par la victime, ce qui se produit dans la moitié des cas environ, c'est généralement que l'accusation a été réduite avant même que le processus judiciaire ait formellement débuté. Ainsi, lorsque des coups ont été effectivement portés ou des blessures infligées, on peut procéder en vertu de l'article 745 plutôt que de porter accusation pour voies de fait. Les juges et les procureurs interviewés nous ont fait remarquer qu'il est plus simple de procéder ainsi; c'est moins dangereux pour la victime qui craint des représailles de la part de son conjoint; c'est moins coûteux pour l'accusé qui, de cette façon, n'écopera pas d'un dossier judiciaire.

\section{b) L'ARRÊT DES PROCÉDURES}

Près de la moitié des causes se terminent avant terme, par un arrêt des procédures. Contrairement à la croyance populaire selon laquelle les femmes retirent leur plainte, on voit que dans la moitié des cas l'arrêt des procédures a été demandé par la défense. Bien sûr, la requête de la défense est parfois basée sur l'absence de la victime en cour mais également sur toutes sortes d'autres considérations : l'accusé a quitté la ville, il ne recommencera pas, un témoin est absent, l'accusé s'est bien comporté depuis le dépôt de la plainte. Les considérations de ce genre expliquent le plus grand nombre d'arrêts de procédures $(45 \%)$. L'absence de preuves vient en second lieu $(34 \%)$ et, enfin, la réconciliation entre les conjoints $(22 \%)$. Fait assez troublant, c'est dans les cas de voies de fait simples et graves qu'on constate le plus de retraits de plaintes. De plus, les femmes qui continuent à vivre avec leur agresseur ont plus tendance à retirer leur plainte.

Tout se passe comme si l'appareil de justice était le plus impuissant à protéger les victimes là où elles sont le plus en danger : dans leur foyer et quand elles sont physiquement agressées.

\section{c) LA JUSTICE SUIT SON COURS}

À la Cour municipale de Montréal, les causes se règlent très vite : dès la première audience dans le tiers des cas; en trois audiences ou moins dans $81 \%$ de ces cas de violence conjugale; c'est-à-dire, le plus souvent, en moins de trois mois. L'arrêt des procédures est une explication car cet arrêt survient assez souvent au début du proces- 
sus; les plaidoyers de culpabilité hâtent également les procédures. Le quart des accusés ont plaidé coupables dès la première audience et $14 \%$ de ceux qui avaient plaidé non coupables ont par la suite changé leur plaidoyer. Il s'ensuit que, sur une population initiale de 367 causes, seulement $13 \%$ d'entre elles $(\mathrm{N}=49)$ ont donné lieu à procès. Ces procès sont très sommaires. À part le témoignage de l'accusé et, moins souvent, de la victime, peu d'éléments de preuve sont présentés. Par exemple, le rapport médical n'a été déposé que dans sept cas.

Ả peine plus de la moitié des causes qui se sont rendues à procès (29 sur 49) ont donné lieu à un verdict de culpabilité. Ce résultat tend à renforcer la position de plusieurs intervenants qui préconisent des chefs d'accusation réduits ou un plus grand recours à l'article 745 afin d'éviter que la femme battue ne subisse une autre défaite. Un non-lieu ou un verdict de non-culpabilité peut, selon certains interlocuteurs, placer la femme dans une position de vulnérabilité accrue.

Dans $53 \%$ des cas, l'accusé s'est tiré indemne de son expérience à la Cour municipale : 177 arrêts des procédures, 12 verdicts de non-culpabilité et 7 non-lieu. On ignore ce qui est advenu de la victime. Les agresseurs condamnés ont reçu des sentences assez clémentes. La majorité $(41 \%)$ se sont vus ordonner de ne pas troubler l'ordre public et d'observer une bonne conduite durant un an. Près du tiers ont bénéficié d'une ordonnance de probation, d'une sentence suspendue ou d'une libération conditionnelle ou non. Le quart a dû payer une amende, généralement de 50 \$. Enfin, une sentence de détention a été rendue dans six cas, en prison provinciale. On ne saurait dire que la justice dissuade les hommes de battre leurs femmes. La gravité de la violence semble avoir peu d'effet sur la décision du juge, ce qui laisse le champ assez libre à l'intimidation : "Si tu ne retires pas ta plainte, je te blesserai davantage.»

\section{DES HOMMES, DES FEMMES}

Les dossiers analysés, les entrevues réalisées, nous laissent l'impression non pas d'une guerre entre les sexes mais de profonds et tragiques malentendus. Les hommes battent les femmes, et rarement l'inverse, parce qu'ils sont plus forts physiquement et socialement. Ils ont les moyens d'exprimer les frustrations, les difficultés de communication, qui sont éprouvées par chacun des conjoints.

Quand la femme est battue, c'est à des hommes qu'elle doit demander de l'aide. C'était le cas, du moins, durant la période étu- 
diée où presque tous les intervenants à la police et à la Cour étaient des hommes.

Or, presque tous les policiers, les avocats de la défense, les procureurs de la Couronne, les juges que nous avons rencontrés, surtout ceux qui avaient plusieurs années d'expérience, ont exprimé des malaises face aux situations de violence conjugale dans lesquels ils jouent un rôle vital. Personne ne sait comment réagir. Personne ne comprend. Personne ne peut s'identifier à la victime mais certains peuvent développer une solidarité lointaine, d'appartenance à un même sexe, avec l'agresseur.

Les appareils policier et judiciaire ne traitent pas les cas de violence conjugale de la même façon que les autres crimes, les données qui précèdent le montrent amplement. Les agressions entre conjoints ne sont justement pas perçues comme des crimes, des atteintes à l'ordre social. Elles sont du ressort privé; de là, une grande partie du malaise éprouvé quand le système pénal doit traiter de situations qui semblent relever du civil, de là, les incohérences relevées; de là, la liberté laissée à la plaignante; de là, l'impunité.

La conclusion logique semble que les femmes doivent prendre plus de pouvoir à l'intérieur du système de justice. Certes, nous ne saurions, comme femmes, dédaigner une plus grande représentativité des femmes. Le problème ne serait pas nécessairement résolu pour autant. Ce profond malentendu entre hommes et femmes dure depuis si longtemps que seule, une contre-tradition d'échanges pourra l'enrayer.

\section{BIBLIOGRAPHIE}

BARIL, M., M.M. COUSINEAU, S. GRA VEL. Le problème des femmes batlues : la police est-elle une solution? Rapport de recherche inédit, Université de Montréal, École de criminologie, 1983.

BARIL, M., M. BEAULIEU, L. LOYER (en préparation). Sile tribunal entendait les femmes battues, Montréal, Centre International de criminologie comparée, Université de Montréal. 